\title{
Applied research deserves Nobel prizes
}

\author{
Nobel prizes for applied research provide recognition for innovations with profound impact, fully in tune \\ with the intentions of Alfred Nobel.
}

The 2009 Nobel Prize in Physics honours discoveries and inventions in optics that have profoundly shaped our daily life ${ }^{1}$. Charles K. Kao won half of the prize for "groundbreaking achievements concerning the transmission of light in fibers for optical communication". The other half of the prize was shared by Willard S. Boyle and George E. Smith for "the invention of an imaging semiconductor circuit - the CCD sensor."

The optical fibre of course is key to the success of the internet, whereas CCD (charge-coupled device) sensors, and more recently complementary metal oxide semiconductor (CMOS) image sensors, are widely used in digital cameras. There is no doubt about the everyday impact of both inventions, and there is no doubt that they deserve a Nobel prize.

Oddly, these breakthroughs were hardly on the minds of commentators ahead of the announcement. Thomson Reuters for example has been predicting Nobel prize winners since 1989, but none of this year's recipients has appeared on any of their lists. Their 2009 predictions included several scientists working in optics, but on more fundamental topics such as quantum optics or metamaterials ${ }^{2}$.

Indeed, this year's award for such applied topics seems to have taken some commentators by surprise. However, there is nothing surprising about rewarding applied science. Indeed, this is fully consistent with Alfred Nobel's testament, where "innovations" are explicitly included. In the translation of the relevant section it reads ${ }^{3}$ : "The whole of my remaining realizable estate [...] shall constitute a fund, the interest on which shall be annually distributed in the form of prizes to those who, during the preceding year, shall have conferred the greatest benefit on mankind. The said interest shall be divided into five equal parts [...] one part to the person who shall have made the most important discovery or invention within the field of physics."

The inclusion of innovations in addition to discoveries is perhaps easily rationalized by Alfred Nobel's own biography. Having learned about the discovery of explosive nitroglycerine, he set out to develop a more stable material that could carry the compound more safely. This investigation led to the discovery of dynamite. The commercialization of dynamite brought Nobel the riches that are the basis of the Nobel foundation. Surely, applied scientific research in the disciplines honoured by a Nobel prize is part of what Nobel describes as benefit to mankind, and the award to applied topics such as optical fibres and CCD sensors certainly fulfils the criteria laid out in the testament.

The recognition of applied research has a long tradition in the awarding of Nobel prizes. Just considering past physics prizes, a number of awards have gone to inventions and discoveries with varying degrees of applied background, such as X-rays (the first Nobel prize, 1901), the transistor (1956), the maser (1964), the integrated circuit (2000), semiconductor lasers (2000) and finally giant magnetoresistance (2007). All recipients received the awards in part for the technological revolution they enabled.

This year's Nobel prize also highlights the importance of industrial research in science. Kao made his discoveries at the Standard Telecommunication Laboratories in the UK, whereas Boyle and Smith invented the CCD chip at Bell Laboratories.

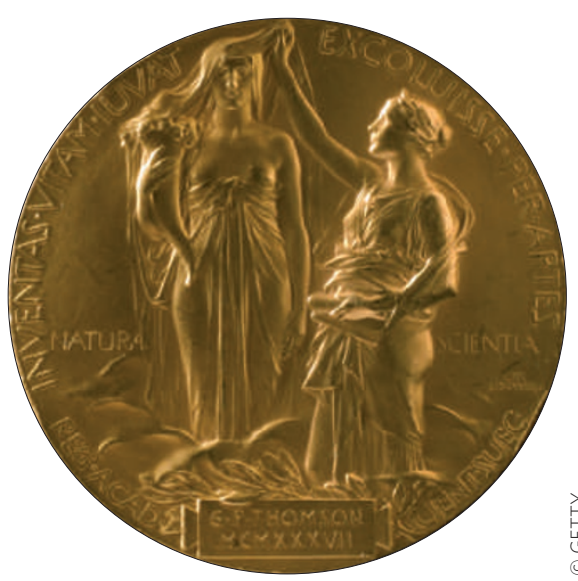

With the steady decline in industrial research in favour of more applied research done in collaboration with universities, the 2009 Nobel Prize in Physics also serves as a reminder that great discoveries do emerge from industrial research. It is the cross-fertilization of scientific insight and technological needs where ingenuity and inventiveness in commercial research can lead to profound breakthrough discoveries. For this reason, this year's Nobel prizes do serve as a stark warning that cuts in corporate research can go too far, and can prevent such innovations from happening.

It is the cross-fertilization

of scientific insight and

technological needs where commercial research

\section{can lead to profound breakthrough discoveries.}

Interestingly, however, even for the more applied prizes the focus of the awards has always been on the initial breakthrough. In case of the 2007 award, for example, Albert Fert and Peter Grünberg received the prize for the discovery of the giant magnetoresistance effect, whereas those researchers whose key inventions enabled commercialization of the effect, for example at IBM, were not acknowledged. Similarly, in addition to Kao's breakthrough in the understanding of optical-fibre materials, advances in fibre design such as core doping with germanium - invented by researchers at Corning - were necessary before optical fibres could be seriously considered for data transmission.

A beautiful summary of the achievements honoured through the Nobel prize, whether applied or fundamental in nature, is the Latin inscription on the Nobel prize medals for physics and chemistry. It is taken from Virgil's Aeneid, sixth verse, line 663: Inventas vitam juvat excoluisse per artes, which loosely translates ${ }^{4}$ as: "And they who bettered life on earth by their newly found mastery." Certainly, many more exciting applied discoveries are awaiting recognition by the Nobel committee. In accordance with Virgil, the cross-section of scientific publications in this issue demonstrates the skills and arts of those performing science at the cutting edge of research.

\footnotetext{
References

1. http://nobelprize.org/nobel_prizes/physics/laureates/2009/

2. http://science.thomsonreuters.com/nobel/nominees/\#physics

3. http://nobelprize.org/alfred_nobel/will/short_testamente.html

4. http://nobelprize.org/nobel_prizes/physics/medal.html
} 